\title{
Relacionamentos familiares de pessoas com transtornos mentais: processos de exclusão e inclusão social
}

Family relationships of people with mental health problems: processes of social exclusion and inclusion

Relaciones familiares de personas con trastornos mentales: procesos de exclusión e inclusión social

\author{
Mariana Moraes Salles'; Sônia Barros"'; Jussara Carvalho dos Santos ${ }^{\prime \prime \prime}$
}

\begin{abstract}
RESUMO
Objetivos: identificar e analisar os processos de exclusão e inclusão social que acontecem na família da pessoa com transtorno mental. Método: utilizou-se a abordagem qualitativa como metodologia de pesquisa. Os sujeitos desta investigação foram 17 usuários de um Centro de Atenção Psicossocial, da cidade de São Paulo, e 12 pessoas de sua rede social. Para a coleta de dados foram realizadas entrevistas semiestruturadas e, para apuração dos dados, foi utilizada a análise do discurso. Resultados: identificou-se que a exclusão social dos usuários na família pode ocorrer pela ruptura e distanciamento ou de uma maneira mais sutil. Porém, na medida em que os usuários são valorizados e encontraram um papel na família, se estabelecem relações de troca e de ajuda mútua. Conclusão: os relacionamentos se constroem em um processo dialético, passando por conflitos e situações de reciprocidade.
\end{abstract}

Descritores: Saúde mental; relações familiares; relações interpessoais; participação social.

\section{ABSTRACT}

Objectives: to identify and to analyze the processes of social exclusion and inclusion that happen in the family of the person with mental disorder. Method: qualitative approach was used as a research methodology. Subjects of this investigation were 17 users of a Psychosocial Care Center, in the city of São Paulo, Brazil, in addition to 12 people from their social networks. For data collection, semi-structured interviews were realizes and, for data analysis, it was the discourse analysis technique. Results: Social exclusion of users of a Psychosocial Care Center in the family can occur due to social disruption and distance, or in a more subtle way. However, to the extent that users are valued and find a role in the family, relationships of exchange and mutual help are established. Conclusion: relationships are developed in a dialectical process, going through conflicts and situations of reciprocity, with the family being the main social inclusion network of these people.

Descriptors: Mental health; family relations; interpersonal relations; social participation.

\section{RESUMEN}

Objetivos: identificar y analizar los procesos de exclusión e inclusión social que ocurren en la familia de la persona con trastorno mental. Método: se utilizó el enfoque cualitativo como metodología de investigación. Las personas de esta investigación fueron 17 usuarios de un Centro de Atención Psicosocial, en la ciudad de São Paulo, Brasil, además de 12 personas de sus redes sociales. Para la recopilación de datos, se realizaron entrevistas semiestructuradas y, para el análisis de datos, fue la técnica de análisis del discurso. Resultados: la exclusión social de los usuarios de un Centro de Atención Psicosocial en la familia puede ocurrir debido a la interrupción social y la distancia, o de una manera más sutil. Sin embargo, en la medida en que los usuarios sean valorados y encuentren un papel en la familia, se establecen relaciones de intercambio y ayuda mutua. Conclusión: las relaciones se desarrollan en un proceso dialéctico, pasando por conflictos y situaciones de reciprocidad, siendo la familia la principal red de inclusión social de estas personas.

Descriptores: Salud mental; relaciones familiares; relaciones interpersonales; participación social.

\section{INTRODUÇÃo}

As relações familiares compõem a principal forma de vínculo social para as pessoas com transtornos mentais, e são, em geral, as pessoas com quem os usuários de Centros de Atenção Psicossocial (CAPS) podem contar, formando a rede de sociabilidade primária.

A família faz parte das microvariáveis do contexto do usuário, influenciando suas possibilidades de participação social. O relacionamento dos familiares com os usuários influencia o processo de reabilitação psicossocial, os familiares podem oferecer suporte para os usuários construírem sua vida cotidiana na comunidade, ou podem causar a sensação de desamparo e solidão.

'Terapeuta Ocupacional. Doutora em Cuidado em Saúde pela Escola de Enfermagem da Universidade de São Paulo. Brasil. E-mail: marianamsalles@gmail.com "Enfermeira. Doutora em Enfermagem. Professora titular da Escola de Enfermagem da Universidade de São Paulo. Brasil. E-mail: sobarros@usp.br

'"Enfermeira. Doutoranda em Cuidado em Saúde pela Escola de Enfermagem da Universidade de São Paulo. Brasil. E-mail: scjussara@gmail.com 
O presente artigo pretende auxiliar no processo reflexivo sobre o papel da família no processo de exclusão e inclusão social da pessoa com transtorno mental, no contexto da Reforma Psiquiátrica, que são aspectos essenciais para a promoção dos cuidados de enfermagem no campo da saúde mental. Assim, os objetivos do presente estudo foram identificar e analisar os processos de exclusão/inclusão social que acontecem a partir do núcleo familiar.

\section{REVISÃO DE LITERATURA}

No modelo centrado no hospital psiquiátrico, com as internações prolongadas e reinternações frequentes, existe uma chance maior dos relacionamentos com os familiares e com outras pessoas da rede social se tornarem frágeis, resultando no isolamento das pessoas com transtornos mentais ${ }^{1-6}$.

Entretanto, com as transformações ocorridas a partir da Reforma Psiquiátrica, ocorreu a redução de leitos psiquiátricos, com o encaminhamento dos usuários para serviços de saúde mental descentralizados e comunitários, redirecionando a oferta de atenção à saúde de um serviço central para um amplo leque de equipamentos de saúde ${ }^{2-7}$. Assim, as famílias das pessoas com transtornos mentais têm se deparado com uma nova forma de tratamento que não é mais centrada na internação em hospital psiquiátrico ${ }^{8}$.

Estas transformações na assistência em saúde mental implicaram ampliação das demandas de comprometimento das famílias da pessoa com transtornos mental, alterando-se significativamente a trajetória de sua participação no processo assistencial. Os familiares se tornaram parceiros fundamentais nos cuidados dos usuários ${ }^{9-10}$, pois podem contribuir com a sentimento de segurança e de apoio dos mesmos ${ }^{11,12}$, além de possibilitarem a realização da assistência em contextos menos estigmatizantes que o hospital psiquiátrico ${ }^{11-14}$.

Porém, os familiares ainda estão em processo de desenvolver um "modo de perceber a pessoa que vivência este adoecer como ser humano integral, multifacetado, histórico e inserido no mundo da vida cotidiana" 15:52. Para os familiares não tem sido um processo muito simples aderir as perspectivas de tratamento preconizadas pelo paradigma psicossocial ${ }^{14}$. Dessa maneira, inserir a família no tratamento da pessoa com transtorno mental tende diminuir as internações, amenizar os conflitos e melhorar a qualidade de vida de todos no domicílio ${ }^{9-11}$.

Neste processo, nota-se a importância de que as famílias sejam ouvidas, que possam compartilhar suas experiências, suas angústias e as vitórias, que possam refletir sobre como é o relacionamento com o familiar que adoeceu e sobre suas estratégias de enfrentamento da doença ${ }^{6}$, tendo como finalidade de diminuir sobrecarga ${ }^{16-21}$. Portanto, investir na rede familiar também abarca a rede social do sujeito, favorecendo a reabilitação psicossocial e a ampliação dos espaços de trocas afetivas e materiais.

\section{METODOLOGIA}

Utilizou-se a abordagem qualitativa como metodologia de pesquisa. Os sujeitos desta investigação foram pessoas com transtorno psíquico, usuários de um CAPS II na região oeste de São Paulo, que estavam vivendo na comunidade e enfrentando o desafio da inclusão social e de construir uma vida significativa, e pessoas de sua rede social.

Foi solicitado ao diretor do CAPS que realizasse uma caracterização do serviço e identificasse ações que têm como objetivo promover a inclusão social. Entre os usuários que participavam dessas ações foram selecionados aqueles: matriculados há, pelo menos, um ano na instituição; conscientes, orientados no espaço e tempo, sem déficit cognitivo moderado ou grave; que concordaram e se dispuseram a participar da pesquisa, e que estiveram presentes nos dias da coleta de dados. Para preservar o anonimato dos participantes estes foram identificados se utilizando a letra $U$ para usuário e a letra $\mathrm{R}$ para pessoas da rede social dos usuários

Também foi solicitado a cada usuário entrevistado que indicasse uma pessoa de sua rede social de relações para participarem da pesquisa, excluindo-se a equipe técnica do CAPS (foram indicados familiares, pessoas de instituições como albergue e igreja, e 1 dos entrevistados indicou um vizinho). Estas pessoas possibilitaram relatos sobre a inclusão social dos usuários na visão da comunidade. O critério de seleção foi a concordância do usuário para a realização da entrevista com a pessoa indicada e o interesse e consentimento da pessoa indicada em participar da entrevista. Foram realizadas no total 29 entrevistas, 17 com usuários e 12 com pessoas de sua rede de relações sociais.

Para a coleta de dados foram realizadas entrevistas semiestruturadas com usuários do CAPS e pessoas que fazem parte da sua rede social. A entrevista semiestruturada se utiliza de perguntas fechadas e abertas, e oferece liberdade para que o entrevistado possa discorrer com liberdade sobre o tema proposto.

O CAPS, cenário do estudo, foi informado sobre os objetivos, finalidades e procedimentos metodológicos do estudo e concordou com o desenvolvimento da pesquisa no local. O projeto foi aprovado também pelo Comitê de Ética e Pesquisa da Secretaria Municipal de Saúde, que autorizou o desenvolvimento da pesquisa (Parecer $n^{\circ} 143 / 08$ ). Os entrevistados assinaram um termo de consentimento livre e esclarecido, autorizando a utilização dos dados coletados na referida pesquisa, e foi garantido sigilo sobre a identidade dos entrevistados. 
Para apuração dos dados foi utilizada a Análise do Discurso, que considera que os discursos estão relacionados com a cultura de uma sociedade, reproduzindo o que um grupo social tem a dizer. Os homens se identificam com o que dizem e nesse processo se constroem enquanto sujeitos ${ }^{22}$. Os discursos devem ser pensados a partir dos processos históricos e sociais que o constituem. O lugar histórico-social em que os sujeitos enunciadores de determinado discurso se encontram envolvem o contexto e a situação em que se encontram, e intervém na produção do discurso.

Após a coleta de dados, as entrevistas foram transcritas na íntegra, constituindo um texto para análise. A partir da leitura repetitiva do texto, os autores se habituaram com os dados, questionando e anotando dados interessantes para a análise. O trabalho de analise de dados ocorre a partir de frases proferidas, delimitando e estabelecendo correspondências entre os fragmentos das frases; neste processo foram identificados padrões, temas analíticos e qualidades discursivas. Uma análise inicial foi realizada no processo de escrita, leitura e análise. A análise foi examinada com colegas do grupo de pesquisa, e após as suas considerações foi conduzida a análise final ${ }^{23,24}$.

Foi identificada a categoria empírica Família: processos de exclusão/inclusão social. Assim, o presente artigo trata dessa categoria empírica com o foco no suporte social oferecido pela família.

\section{RESULTADOS}

\section{Família: processos de exclusão social}

É comum em qualquer família ocorrerem conflitos e tensões, o mesmo ocorre nas famílias de pessoas com transtornos mentais. Porém, quando os conflitos familiares se tornam exacerbados e mais difíceis de serem resolvidos, existe uma possibilidade maior de ocorrer a exclusão social da pessoa com transtorno mental.

Uma das formas de exclusão social vivenciada pelas pessoas com transtornos mentais é o distanciamento dos familiares, que se retiram do contato social com o usuário, abandonando a relação. Desta forma, os familiares deixam de ser uma referência para o usuário, uma pessoa com quem podem contar ou manter uma relação de troca afetiva. $\mathrm{O}$ distanciamento pode ocorrer com a diminuição da frequência dos encontros com a pessoa com transtorno mental, com contatos que se tornam espaçados, como relatado a seguir.

O pai é uma figura mais distante. Não é uma pessoa que participa da vida dele. (R13)

Outra forma de distanciamento familiar pode ocorrer pela superficialidade do contato, que é condizente com a ideologia de preconceito e desvalorização do usuário, como exemplificado por pessoas da rede social do usuário. Esta forma de relacionamento também é uma forma de reprodução do contexto social, de uma sociedade pouco solidária, em que as pessoas podem valorizar mais o seu trabalho, por exemplo, do que sua rede de relações sociais.

E com outras pessoas, com a minha irmã, por exemplo, ele tem uma convivência superficial. Porque, ele tem um maior contato comigo. Com as primas é só 'Oi, tudo bem, como vai?', e só. (R8)

Frente as dificuldades de relacionamentos e à falta de compreensão sobre como lidar com uma pessoa com transtorno mental, muitas vezes o distanciamento da família em relação ao usuário se dá explicitamente, na forma de uma ruptura.

O outro filho que eu tenho. Quando o M. começou a aparecer com essa doença, ele simplesmente mudou de casa e se afastou muito da família, até desaparecer mesmo. (R4)

Então é difícil, a convivência com os irmãos é complicada, eles não se entendem. Ele [usuário] prefere ficar nessa situação [morar em albergue] do que viver com os irmãos. (R8)

Outra dificuldade que a família enfrenta é a sobrecarga que sofrem devido à presença de uma pessoa com transtorno mental. Os familiares se sentem sobrecarregados em todos os sentidos: físico, financeiro, demanda de tempo, e a sobrecarga emocional de quem vive o dia a dia com a pessoa e investe seus esforços em tentar ajudá-la a se desenvolver.

[Sobre relacionamento com usuário] Eu estou cansada, eu estou ficando para lá do estresse. Porque ele é uma pessoa que suga todas as energias. (R15)

Porque com dinheiro ou sem dinheiro, tem que segurar a barra dele. Eu não posso abandonar ele. (R7)

As formas de exclusão social das pessoas com transtornos mentais na família podem se dar de uma maneira objetiva, pela ruptura, distanciamento e falta de apoio, ou de uma maneira subjetiva, pelas formas como a relação é construída, em um contato permeado por sentimentos de medo, receio, cansaço, superproteção e sobrecarga. Assim, a família mostra que, muitas vezes, precisa de ajuda dos profissionais da saúde para favorecer a inclusão social do usuário.

Além disso, é importante não culpar a família por sua forma de se relacionar com a pessoa com transtorno mental, mas compreender a complexidade da atenção em saúde mental e o papel central que os familiares ocupam neste processo. 


\section{Família: processos de inclusão social}

Por outro lado, também foi possível identificar diversos avanços em relação às possibilidades de inclusão social que ocorrem a partir do núcleo familiar. Apesar dos conflitos enfrentados no relacionamento do dia a dia, os familiares têm mostrado seu potencial em ajudar e estabelecer relações de trocas com os usuários.

O acolhimento da família é um dos aspectos importantes na inclusão social da pessoa com transtorno mental, pois ao serem acolhidos eles podem sentir que pertencem a um grupo social, rompendo com o isolamento que é um dos caminhos que leva à exclusão social.

É porque eu tenho uma família acolhedora, porque se eu não tivesse uma família, família de verdade, que é a coisa principal que tem... Se por ventura eu não tivesse uma família assim, talvez, eu estaria louco. (U10)

Outro aspecto importante em relação aos familiares é que eles auxiliam os usuários a enfrentarem o preconceito. Eles têm o potencial de emprestar o seu poder contratual ao usuário, e oferecer um suporte para que ele circule na sociedade e estabeleça vínculos não apenas com a família. Se os familiares conseguem romper o preconceito em relação a doença mental no seu relacionamento com os usuários, essa nova forma de lidar com a pessoa começa a aparecer na sociedade, servindo como um modelo. O núcleo familiar pode ser um ponto de partida para os usuários enfrentarem o preconceito em relação a doença mental e construírem o seu caminho em direção a inclusão social.

E junto com o meu marido, eu fui capaz de mostrar a cara e mostrar o meu filho para o mundo. Que ele era doente, mas não era diferente dos outros. Que ele podia estar em qualquer lugar. (R4)

Também foi identificado que um dos suportes oferecidos pelos familiares é o apoio financeiro. No contexto, em que a maioria das pessoas com transtornos mentais vive uma realidade de desvantagem financeira, muitas vezes os familiares precisam auxiliar os usuários para que eles tenham acesso às condições materiais mínimas.

Apesar de a literatura enfatizar a sobrecarga da família que precisa auxiliar financeiramente a pessoa com transtorno mental ${ }^{10}$, por outro lado, no presente trabalho os discursos também apontam o lado positivo desta ajuda dos familiares, que é uma forma de suporte social e inclusão do usuário no núcleo familiar.

[Sobre ajuda material] Normalmente é meu sobrinho médico. E minha irmã também. (U10)

A minha irmã sempre me ajuda bastante. Às vezes ela compra o que precisa. (U11)

A relação com os familiares também foi apontada como importante para ajudar as pessoas com transtornos mentais a circularem pela cidade. Os familiares oferecem companhia para os usuários frequentarem diferentes espaços sociais, um suporte fundamental que ajuda as pessoas com transtornos mentais a interagir e participar dos ambientes sociais, auxiliando no processo de inclusão social desta população.

Eu saio com a minha mãe mesmo... vamos à igreja e vem para o CAPS. (U13)

Eu converso com ela [tia], ou saio com ela. Nós fomos pra praia um dia, eu viajei com ela. (U8)

Além disso, o tratamento dos usuários no CAPS também auxilia os familiares a construírem seu relacionamento com a pessoa com transtorno mental, pois eles recebem orientações da equipe técnica e dividem com a instituição responsabilidades que aliviam a sobrecarga familiar. O fato dos usuários não terem o dia centrado unicamente na família, mas ter outras opções de atividades e contatos sociais é um fator que auxilia o relacionamento com os familiares nos horários que estão juntos.

E dá uma aliviada para família também, porque fica todo mundo muito estressado quando a pessoa fica o dia inteiro, precisa dar um tempinho, sabe. (R15)

Melhorou um pouquinho [o relacionamento com a família] porque a psicóloga tem orientado ela. (U6)

Neste contexto em que os usuários ganham mais autonomia, são valorizados e empoderados, é possível que eles encontrem um papel social no núcleo familiar, uma função social que lhes pertence, um lugar confortável na dinâmica familiar. Se a pessoa com transtorno mental não é vista como incapaz, ela tem a oportunidade de encontrar uma nova identidade na família, que não apenas a identidade de pessoa doente que precisa ser cuidada.

Então nessa construção [da casa], eu incluí muito o R. E eu me acostumei a ter o R. assim comigo, as opiniões dele são tão boas. Veja, eu não imaginava. Foi uma das coisas mais lindas que já me aconteceu. (R12)

Muitas vezes eu preciso ir viajar, e ele se propõe a ficar na casa e cuidar dos cachorros. Então ele gosta de ajudar. Então ele se sente útil, então eu acho que aquilo faz ele feliz, ele se sente importante. (R8)

É preciso ressaltar que uma das principais questões sobre as relações familiares é como se dá esta relação, e é claro que muitas vezes o usuário vai precisar de ajuda e cuidados do seu familiar e a disponibilidade das famílias para ajudá-los é um aspecto positivo. Porém, é preciso estar atento para que esta relação não retire do usuário valor e poder contratual, que ele não seja visto com piedade e como alguém que deve apenas ser cuidado. 


\section{DISCUSSÃo}

O transtorno mental é um fenômeno pouco compreendido e aceito e, muitas vezes, temido pelos familiares ${ }^{8}$, que relatam dificuldades trazidas pela convivência com a pessoa com transtorno mental. Eles refletem sobre a falta de tempo, falta de conhecimento específico sobre o transtorno mental, sobre a dificuldade em lidar com a situação de crise e com as transformações ocorridas na dinâmica familiar após a situação de adoecimento, o que converge com a literatura científica sobre o tema ${ }^{8-21}$.

Os familiares relatam sentimentos referentes à sobrecarga que o papel do cuidador thes confere, e ao mesmo tempo, denotam afeto pelo familiar adoecido. Eles relatam atitudes que vão da disponibilidade para o acolhimento até o cansaço extremo por excesso de demandas da pessoa com transtorno mental, caracterizando a sobrecarga largamente discutida por diversos autores ${ }^{16-21 ; 25}$.

Neste processo dialético de exclusão e inclusão social, os familiares também podem considerar recompensador cuidar da pessoa com transtorno mental ao vê-la melhorando e se estabelecendo na comunidade, assim, familiares e usuários podem desenvolver um relacionamento confortável, solidário e amoroso ${ }^{26}$, promovendo, assim, a reabilitação psicossocial.

O relacionamento com familiares é algo estabelecido ao longo do tempo, construído a partir da convivência no cotidiano. A história do sujeito é entrelaçada com história das pessoas de sua rede social, com diversos momentos em que a presença das pessoas da família foi marcante positiva ou negativamente.

A vida cotidiana é a arena da vida em família, onde os familiares se conectam a partir das atividades da vida do dia a dia ${ }^{27}$. A constituição da família, enquanto rede social particular do sujeito, possibilita também a organização social, pois é partir da família que se constituem núcleos de moradia, que se estrutura a educação infantil e, muitas vezes, que se oferecem os serviços de saúde públicos.

Na medida em que os usuários são valorizados e encontraram um papel na família, as relações com os familiares se tornam relações de troca, de ajuda mútua, rompendo com a dinâmica do cuidador e daquele que deve ser cuidado. O usuário e sua família se apoiam reciprocamente e podem dividir os problemas da vida. A pessoa com transtorno mental deixa de ser um peso constante para o familiar, construindo relações benéficas tanto para o usuário como para as pessoas de sua família, quebrando o ciclo de isolamento e segregação.

A inclusão social passa por deixar de ser uma pessoa que só recebe, um caso de caridade, para tornar-se um membro ativo da sociedade, que pode contribuir com os outros ${ }^{27}$. Neste cenário, a pessoa com transtorno mental passa a ser um cidadão com direito à participação social na cidade onde vive com sua família e amigos ${ }^{4}$.

Entretanto, muitas vezes as famílias necessitam de um suporte social organizado para que consigam articular recursos internos para o enfrentamento de situações relacionadas ao transtorno menta ${ }^{28,29}$. Neste contexto, quanto maior o número de horas junto com os usuários, maior o nível de sobrecarga percebido pelos cuidadores. Quando os usuários saem de casa e frequenta um programa de atenção diária, que os ajudam a lidar com problemas do dia a dia e melhoram sua autonomia e habilidades sociais ${ }^{29}$, os familiares se sentem menos sobrecarregados ${ }^{16-21}$. Desta maneira, os enfermeiros e demais profissionais dos serviços de saúde devem estar atentos à promoção de abordagens que seguem a lógica supracitada, além de oferecer a escuta terapêutica ${ }^{30}$ aos familiares a fim de contribuir com a diminuição da sobrecarga diária.

Assim, é preciso que os enfermeiros e as equipes dos serviços sejam preparados para ter como objeto do trabalho não apenas a pessoa adoecida, mas, devido mudança paradigmática que vem ocorrendo na área de saúde mental, é urgente que o foco da atenção seja ampliado, incluindo a família como elemento central no atendimento em saúde mental ${ }^{15 ; 30}$.

\section{CONCLUSÃO}

Os familiares estão impregnados pelos valores do senso comum, construídos historicamente, que colocam a pessoa com transtorno mental em um lugar de desvalorização e incapacidade. Com o início da doença estes valores do senso comum influenciam as relações familiares.

Porém, apesar das dificuldades de relacionamento com os familiares, a família também foi apontada como a principal rede social de suporte desta população. Percebe-se uma contradição em relação à família que, ao mesmo tempo em que pode excluir, também representa um dos principais apoios para a pessoa com transtorno mental em seu processo de inclusão social.

$\mathrm{Na}$ atenção à saúde de pessoas com transtornos mentais é preciso auxiliar estas pessoas para que elas consigam aumentar seu poder contratual em todos os locais em que circulam, começando pelo próprio núcleo familiar. É possível que transformando as formas de relacionamento com os sujeitos com transtorno mental, se alcance uma mudança na forma de inclusão social condizente com o atual processo de reforma psiquiátrica. 


\section{REFERÊNCIAS}

1. Salles MM, Barros S. Exclusión/inclusión social de usuarios de un centro de atención psicosocial en la vida cotidiana. Texto contexto-enferm. 2013 [cited 2019 Oct 10]; 22: 704-12. DOI: http://dx.doi.org/10.1590/S0104-07072013000300017

2. Pitta AMF, Guljor AP. A violência da contrarreforma psiquiátrica no Brasil: um ataque à democracia em tempos de luta pelos direitos humanos e justiça social. Cadernos do CEAS: Revista Crítica de Humanidades. 2019 [cited 2019 Oct 10]; 246:6-1. DOI: https://doi.org/10.25247/2447-861X.2019.n246.p6-14

3. Amarante $P$, Nunes MDO. A reforma psiquiátrica no SUS e a luta por uma sociedade sem manicômios. Ciência \& Saúde Coletiva. 2018 [cited 2019 Oct 10]; 23(6), 2067-74. DOI: http://dx.doi.org/10.1590/1413-81232018236.07082018

4. Almeida RLM de, Pereira HMB, Silva SBIM, Magno C de O, Ferreira AP. Impacto da Desinstitucionalização na Qualidade de Vida de Indivíduos com Transtornos Psiquiátricos. REAS [Internet]. 2019 [cited 2019 Oct 10]; 11(15):e1233. DOI: https://doi.org/10.25248/reas.e1233.2019

5. Amarante P, Torre EHG. "Back to the city, Mr. citizen!" - psychiatric reform and social participation: from institutional isolation to the anti-asylum movement. Revista de Administração Pública. 2018 [cited 2019 Oct 10]; 52(6), 1090-1107. DOI: https://dx.doi.org/10.1590/0034-761220170130

6. Franco RF, Stralen CJV. Psychiatric deinstitutionalization: from confinement to inhabiting in the city of belo horizonte. Psicol Soc, 2015 [cited 2019 Oct 10]; 27:312-21. DOI: http://dx.doi.org/10.1590/1807-03102015v27n2p312

7. Santos JC, Barros S, Huxley PJ. Social inclusion of the people with mental health issues: Compare international results. International Journal of Social Psychiatry. 2018; [cited 2019 Nov 04] 64(4):344-50. DOI: 10.1177/0020764018763941

8. Colveiro LA, Ide CAC, Rolim MA. Family and mental disease: the hard living with the differences. Rev Esc Enferm USP. 2004 [cited 2019 Nov 04]; 38:197-205. DOI: http://dx.doi.org/10.1590/S0080-62342004000200011

9. Reis TL, DahI CM, Barbosa SM, Teixeira MR, Delgado, PGG. Burden and participation of family in the care of Psychosocial Care Centers users. Saúde Debate. 2016 [cited 2019 Nov 04]; 40(109):70-85. DOI: http://dx.doi.org/10.1590/0103-1104201610906

10. Carvalho PAL, Moura MS, Carvalho VT, Reis MCS, Lima CBO, Sena ELS. The family in the psychosocial rehabilitation of people with mental suffering. J Nurs UFPE on line. 2016 [cited 2019 Nov 04]; 10(5):1701-8. Available from: https://periodicos.ufpe.br/revistas/revistaenfermagem/article/view/13545

11. Randemark NFR, Barros S. The family in therapeutic design of users of CAPS: Representations of health professionals. J Nurs UFPE on line. 2014 [cited 2019 Nov 04]; 8(7):1956-64. Available from: https://periodicos.ufpe.br/revistas/revistaenfermagem/article/view/9871

12. Gomes TB, Santos JBF. Dilemmas and setbacks of families in social vulnerability in the context of psychiatric deinstitutionalization. Physis. 2016 [cited 2019 Nov 04]; 26(1):271-287. DOI: http://dx.doi.org/10.1590/S010373312016000100015

13. Oliveira KKD, Rangel CT, Maia CAAS, Júnior JMP, Fernandes RL, Miranda FAN. Contextual aspects of family participation in psychosocial care centers. J Nurs UFPE on line. 2016 [cited 2019 Nov 04]; 10(4):3676-81. Available from: https://periodicos.ufpe.br/revistas/revistaenfermagem/article/view/11143/12647

14. Dimenstein M, Sales AL, Galvão E, Severo AK. The Psychosocial Care Strategy and family participation in mental health care. Physis. 2010 [cited 2019 Nov 04]; 20:1209-26. DOI: http://dx.doi.org/10.1590/S0103-73312010000400008

15. Cavalheri SC. Changes in mental health care model and the impact on family. Rev Bras Enferm. 2010 [cited 2019 Nov 04]; 63:517. DOI: http://dx.doi.org/10.1590/S0034-71672010000100009

16. Delgado PG. Family burden, support groups and coping style among family members of patients in Psychosocial Care Centers. Physis. 2014 [cited 2019 Nov 04]; 24(4):1103-1126. http://dx.doi.org/10.1590/S0103-73312014000400007

17. Alves JFM, Almeida AL, Mata MAPD, Pimentel MH. Problems of caregivers of patients with schizophrenia: The family burden. Revista Portuguesa de Enfermagem de Saúde Mental. 2018 [cited 2019 Nov 04]; (19), 8-16. DOI: http://dx.doi.org/10.19131/rpesm.0197

18. Mota SD, Pegoraro RF. Conceptions of family on a psychosocial care center. Revista Pesquisas e Práticas Psicossociais. 2018 [cited 2019 Nov 04]; 13(2), 1-17. Available from: http://pepsic.bvsalud.org/scielo.php?script=sci_arttext\&pid=S1809$89082018000200006 \& \operatorname{lng}=$ en $\&$ nrm $=$ iso

19. Ramos AC, Calais SL, Zotesso MC. Convivência do familiar cuidador junto a pessoa com transtorno mental. Contextos Clínicos. 2019 [cited 2019 Nov 04]; 12(1), 282-302. DOI: http://dx.doi.org/10.4013/ctc.2019.121.12

20. Soares MH, da Costa Farinasso AL, de Souza-Gonçalves C, Machado FP, Mariano LKFR, dos Santos CD. Overload and satisfaction of family members of patients with schizophrenia. Cogitare Enfermagem. 2019 [cited 2019 Nov 04];24. DOI: http://dx.doi.org/10.5380/ce.v24i0.54729

21. Nascimento KC, Kolhs M, Solange M, Berra E, Olschowsky A, Guimarães AN. The family challenge in for people care suffering from mental disorder. Revi Enferm UFPE on line. 2016 [cited 2019 Nov 04]; 10:940-48. Available from: https://www.lume.ufrgs.br/bitstream/handle/10183/141077/000990845.pdf?sequence=1

22. Vianna PCM, Barros S. Discourse analysis: a theoretical review. REME Rev Min Enferm. 2003 [cited 2019 Nov 04]; 7:56-60. Available from: https://www.reme.org.br/artigo/detalhes/785

23. Shaw S, Baile J. Discourse analysis: what is it and why is it relevant to family practice? Fam Prac. 2009 [cited 2019 Nov 04]; 26:413-19. Available from: https://www.ncbi.nlm.nih.gov/pmc/articles/PMC2743732/

24. Mazière F. A análise do discurso: história e práticas. São Paulo: Parábola; 2007.

25. Borba LO, Schwartz E, Kantorski LP. Stress on families living with the reality of mental disorder. Acta Paul Enferm. 2008 [cited 2019 Nov 04]; 21:588-94. DOI: http://dx.doi.org/10.1590/S0103-21002008000400009 
26. Gray B, Robinson CA, Seddon D, Roberts A. An emotive subject: insights from social, voluntary and healthcare professionals into the feelings of family cares for people with mental health problems. Health Soc Care Community. 2009 [cited 2019 Nov 04]; 17:125-32. DOI: https://doi.org/10.1111/j.1365-2524.2008.00803.x

27. Davidson L, Stayner DA, Nickou C, Stryron TH, Rowe M, Chinman ML. "Simply to let be in": Inclusion as basis for recovery. Psychiatr Rehabil J. 2001 [cited 2019 Nov 04]; 24:375-88. DOI: http://dx.doi.org/10.1037/h0095067

28. Koukia E, Medianos MG. Is psychosocial rehabilitation of schizophrenic patients preventing family burden? A comparative study. J Psychiatr Ment Health Nurs. 2005 [cited 2019 Nov 04]; 12:415-22. DOI: https://doi.org/10.1111/j.13652850.2005.00852.x

29. Lima MS, Aguiar ACL, Sousa MM. The shared care in mental health as potential of user autonomy. Psicol Estud. 2015 [cited 2019 Nov 04]; 20(4):675-686. DOI: http://dx.doi.org/10.4025/psicolestud.v20i4.28309

30. Andrade JNB, Siqueira FM. A atuação do enfermeiro nos Centros de Atenção Psicossocial. Rev Enferm UFJF [Internet]. 2018 [cited 2019 Jan 21]; 4(1):83-92. Available from: https://periodicos.ufjf.br/index.php/enfermagem/article/view/14020 